\title{
El portafolio como herramienta didáctica: un estudio en escuelas universitarias de enfermería
}

\author{
M.C. Canalejas-Pérez
}

Objetivos. Describir las experiencias con portafolio que se están llevando a cabo en las escuelas de enfermería españolas y analizar la opinión del profesorado con experiencia en el empleo del portafolio, sobre su utilidad como instrumento de aprendizaje y/o evaluación. Sujetos y métodos. Estudio descriptivo transversal realizado durante los meses de abril a junio de 2008. Participaron 58 profesores de las escuelas de enfermería españolas, con experiencia en la utilización del portafolio en la formación de enfermeras. Los datos se recogieron mediante un cuestionario elaborado para tal fin y se estudiaron las variables: perfil del profesorado, experiencias con portafolio y opinión sobre la utilidad de dicha herramienta. Resultados. La mayor parte de las experiencias se llevan realizando entre uno y tres años, principalmente en los periodos de aprendizaje teórico. Los portafolios constan de trabajos diseñados por el profesor y trabajos elegidos libremente por los estudiantes, en los que destaca la escritura reflexiva. Como ventajas resaltaron que el portafolio potencia la capacidad de reflexión y el pensamiento crítico, permitiendo identificar los puntos fuertes y débiles del estudiante. Como inconvenientes destacaron principalmente el incremento del trabajo del profesor y del estudiante. Conclusiones. El portafolio es una herramienta que se está introduciendo de manera progresiva en la formación de enfermeras. Su utilización contribuye al aprendizaje significativo de las competencias, con un enfoque reflexivo y crítico, y permite descubrir las diferentes rutas que siguen los estudiantes en la adquisición de aquéllas.

Palabras clave. Aprendizaje activo. Educación basada en competencias. Evaluación formativa. Investigación en educación de enfermería. Portafolio. The portfolio as an educational tool:
a study in schools of nursing

Aims. To describe the experiences with portfolios which are be- ing developed in the Spanish Schools of Nursing, and to analyze the opinion of teachers with experience in the use of portfolios on its use as a learning and/or assessment tool. Subjects and methods. Descriptive transversal study carried out from April to June 2008 with the participation of 58 teachers of the Spanish Schools of Nursing, who have a wide experience in the use of the portfolio for training nurses. The data were collected through a questionnaire created for it and the following aspects were studied: teachers' profile, experience with portfolios and opinion on the use of such tool. Results. Most of the experiences are being carried out for 1 to 3 years, mostly in theoretical learning periods. Portfolios are composed of works designed by the teacher and works freely chosen by the students, among which we must highlight thoughtful writing. One of the advantages they stressed was that the portfolio improves thinking ability and critical thought, allowing us to identify student's strengths and weaknesses. The main disadvantage they found was the increase of teacher and student's work. Conclusions. The portfolio is a tool which is being brought into the training of nurses gradually. Its use contributes the significant learning of the competences, with a thoughtful and critical approach, and allows us to discover the different steps followed by the students in their acquisition.

Key words. Active learning. Competency-based education. Formative assessment. Nursing education research. Portfolio.

\section{Introducción}

Con el proceso de convergencia europea de la educación superior, los escenarios y las metodologías de la enseñanza universitaria deben experimentar una profunda renovación, de manera que reviertan en un aprendizaje más responsable y activo por parte de los estudiantes. Asimismo, es necesario buscar estrategias de evaluación que se
Escuela Universitaria de Enfermería La Paz. Universidad Autónoma de Madrid. Madrid, España.

Correspondencia Prof. María del Coro Canalejas Pérez. Escuela Universitaria de Enfermería La Paz. Universidad Autónoma de Madrid. Paseo de la Castellana, 261. E-28046 Madrid.

Fax +34 917277032

E-mail

ccanalejas.hulp@salud. madrid.org

Agradecimientos A todos los profesores y profesoras que han participado en el estudio, porque sin ellos este trabajo no tendría contenido; a A. Rodríguez Marcos, R.M. Esteban Moreno y M.L. Martínez Martín, por su apoyo y sabios consejos. 


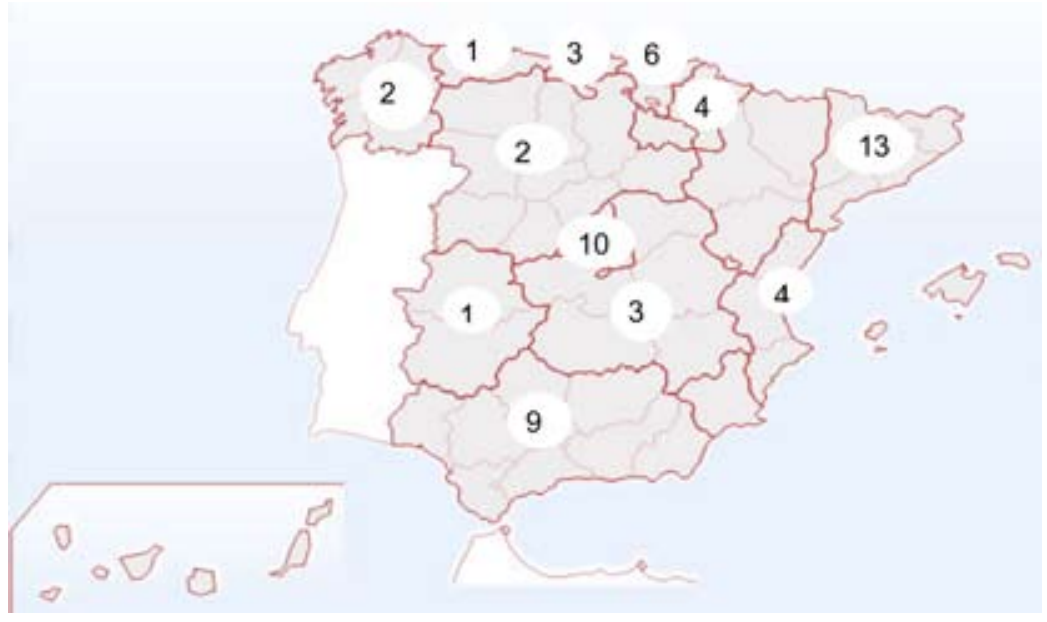

Figura 1. Distribución del profesorado que ha participado.

alejen del enfoque derivado de la tradición cuantitativa y contribuyan a potenciar la capacidad de autorregulación del estudiante.

Una técnica didáctica, acorde con estos planteamientos, que se ha ido introduciendo a lo largo de los últimos años es el portafolio. Aunque el concepto de portafolio del estudiante varía en los diferentes autores, siguiendo a Cano [1] y Klenowski [2], se puede considerar como una selección de muestras del trabajo de un estudiante que, realizadas mediante la escritura y el diálogo reflexivo, reflejan la historia de sus esfuerzos, progresos y logros alcanzados en una área determinada, constituyendo en definitiva, como afirma Martin-Kniep [3], 'el espejo de una realidad en evolución'.

En el contexto educativo, el portafolio se está utilizando no sólo para potenciar el aprendizaje y evaluar el proceso de adquisición de competencias de los estudiantes [2] en diversas disciplinas, sino que también es una importante fuente de evidencia de desarrollo personal y profesional para los docentes. Desde el año 1992, en que Glen y Hight [4] publicaron uno de los primeros artículos relativos a su utilización en la formación de enfermeras, los estudios que analizan su utilidad como instrumento de aprendizaje y evaluación en dicha formación se han incrementado de manera progresiva hasta la actualidad. En la literatura existente al respecto se recoge principalmente la opinión de los estudiantes [5-8], pero sólo se ha encontrado un estudio [9] encaminado a determinar la percepción del profe- sorado sobre el uso del portafolio. Por esta razón, se ha estimado oportuno describir las experiencias con portafolio que se están llevando a cabo en las escuelas de enfermería españolas y analizar la opinión del profesorado con experiencia en el empleo del portafolio, sobre su utilidad como instrumento de aprendizaje y evaluación.

\section{Sujetos y métodos}

\section{Diseño}

Estudio descriptivo de corte transversal.

\section{Ámbito de estudio}

Las 107 escuelas universitarias de enfermería de España.

\section{Sujetos}

Todos los profesores de las escuelas de enfermería de España que habían utilizado o estaban utilizando el portafolio como instrumento de aprendizaje y/o evaluación.

\section{Variables}

- Perfil del profesorado con experiencia en la utili- 
zación del portafolio: edad, género y experiencia docente.

- Experiencias realizadas con el portafolio: tiempo que se lleva utilizando, materia y área de aprendizaje en la que se emplea, número de alumnos que lo elaboran, concepto de portafolio que orienta las experiencias, componentes y evaluación del portafolio.

- Opinión del profesorado sobre ventajas e inconvenientes del portafolio como instrumento de aprendizaje y evaluación.

\section{Procedimientos}

\section{Selección del profesorado}

Se contactó por teléfono y/o correo electrónico con el equipo de dirección de las escuelas de enfermería, con el fin de solicitar información sobre posibles experiencias relacionadas con el portafolio.

\section{Elaboración de la herramienta de recogida de datos}

Para recoger la opinión del profesorado se diseñó un cuestionario que constaba de los siguientes apartados:

- Perfil del profesorado: edad, género, formación.

- Experiencia llevada a cabo con el portafolio: asignatura, curso, número de estudiantes matriculados, número de estudiantes que habían elaborado el portafolio, tipo de portafolio, número de años que se estaba llevando a cabo la experiencia, concepto y componentes del portafolio, forma de evaluación de éste.

- Opinión del profesorado sobre las ventajas y dificultades de la utilización del portafolio: este apartado constaba de 39 ítems que contenían afirmaciones sobre esta herramienta. Para valorar el grado de acuerdo del profesorado con dichos ítems, se utilizó una escala de respuesta Likert de cuatro puntos: 'nada' (1), 'poco' (2), 'bastante' (3) y 'mucho' (4). Al final del cuestionario se destinó un espacio de texto libre para posibles comentarios que desearan realizar sobre dicha experiencia.

El contenido del cuestionario fue evaluado por dos profesoras expertas de la Universidad Autónoma de Madrid. Asimismo se valoró mediante un estudio piloto con ocho profesores, cuatro sin experiencia y cuatro con experiencia en la utilización del portafolio.

\section{Recogida de datos}

El cuestionario se envió por correo electrónico, en los meses de abril a junio de 2008, adjuntando una carta de presentación que informaba a las profesoras/es sobre el estudio y aseguraba el anonimato y la confidencialidad de los datos. La recepción del cuestionario cumplimentado implicaba el consentimiento a participar en el estudio.

\section{Análisis de datos}

Los datos cuantitativos se analizaron con técnicas estadísticas descriptivas mediante el programa SPSS v. 15.0 y los datos cualitativos se sometieron a la técnica de análisis de contenido, definiendo categorías y subcategorías por consenso interjueces.

\section{Resultados}

Se recibió información de 101 escuelas (95\%), localizándose profesores con experiencia en la utilización del portafolio en 33 (34\%) de ellas. De los 69 profesores localizados, 58 (84\%) enviaron el cuestionario cumplimentado (Fig. 1).

\section{Perfil del profesorado}

De los 58 profesores que constituyen la muestra, 47 son mujeres y 11 son hombres, con una edad media comprendida principalmente entre 51 y 60 años. El 64\% $(n=37)$ del profesorado lleva más de 15 años trabajando en la docencia.

\section{Experiencias que se están realizando con portafolio}

\section{Descripción de las experiencias}

De las 72 experiencias con portafolio descritas por los profesores, 60 (84\%) se llevan realizando entre uno y tres años, distribuyéndose mayoritariamente durante los periodos de aprendizaje teórico (76\%). Las materias en que se utiliza el portafolio con más frecuencia son: Fundamentos de Enfermería (17\%), Enfermería Comunitaria (13\%), Ciencias Psicosociales Aplicadas (10\%), Enfermería Medicoquirúrgica y Administración de los Servicios de Enfermería (7\%).

El profesorado realiza el seguimiento de los estudiantes mediante tutorías programadas y a demanda, que oscilan entre una y cinco por es- 


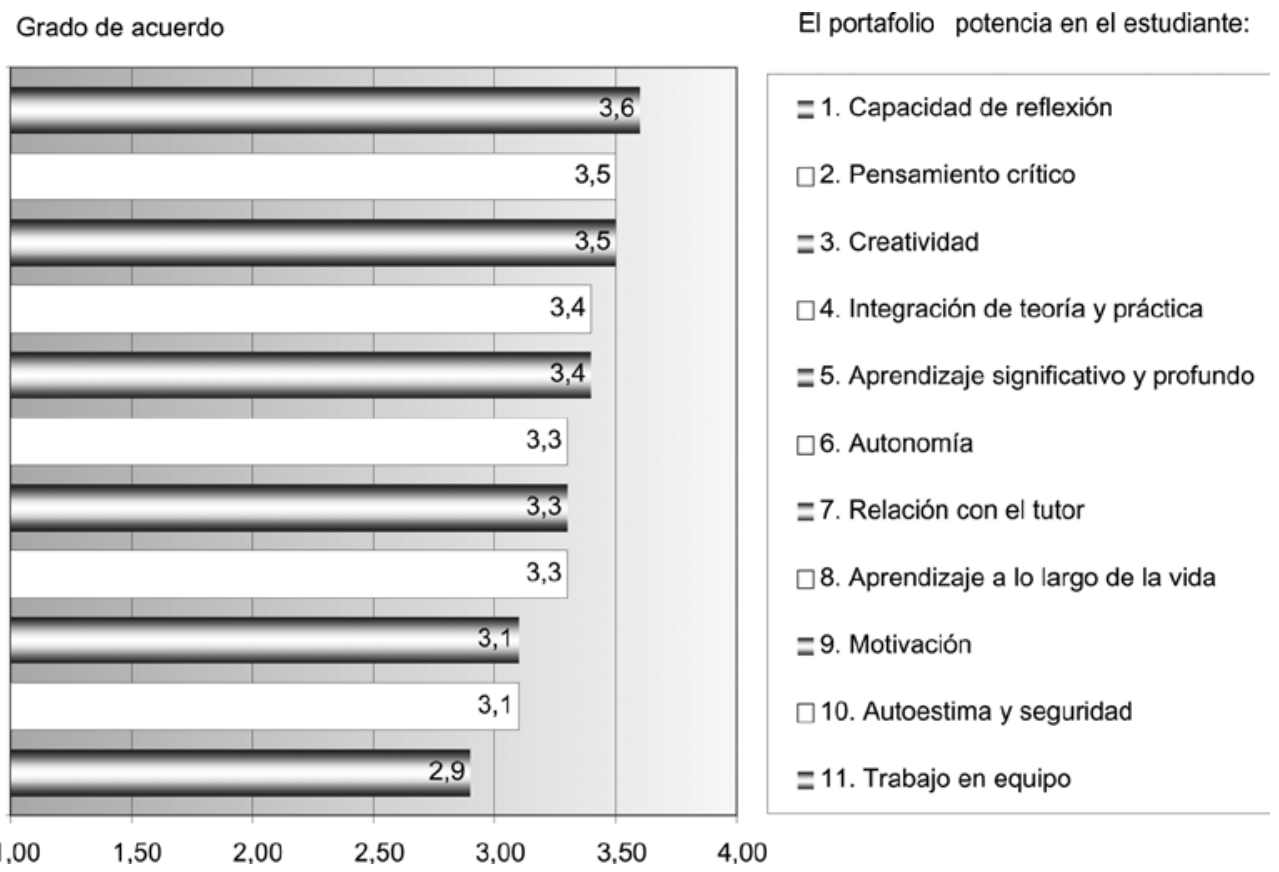

Figura 2. Opinión del profesorado sobre las ventajas del portafolio como instrumento de aprendizaje. Escala Likert: nada, 1; poco, 2; bastante, 3; mucho, 4.

tudiante. Para la evaluación final, en 50 (70\%) experiencias se adjudica al portafolio entre el 10 y el $50 \%$ del conjunto de la nota de las asignaturas.

\section{Concepto de portafolio que orienta las experiencias}

El profesorado coincide en considerar el portafolio como una recopilación de producciones del estudiante. En 36 (51\%) casos, parte de esos trabajos han sido diseñados por el profesor y parte constituyen evidencias seleccionadas libremente por el estudiante; en 19 (27\%), todos los trabajos han sido diseñados por el docente, y en 8 (11\%) el portafolio contiene sólo evidencias seleccionadas por el estudiante. En 26 casos (36\%) resaltan que el portafolio incluye la autoevaluación.

\section{Tipo de portafolio y número}

\section{de alumnos que lo elaboran}

En 50 (70\%) experiencias han realizado el portafolio más de 50 estudiantes, predominando la elaboración individual (74 \%) frente a la realización en grupo.

\section{Componentes de los portafolios}

Como componentes obligatorios del portafolio se incluye el diario reflexivo en 48 (66\%) experiencias, los trabajos realizados por el estudiante en 47 (65\%), la autoevaluación en 42 (59\%), los comentarios del tutor en 37 (51\%) y los comentarios críticos de las fuentes bibliográficas utilizadas en 32 (45\%). Sólo 14 profesores han resaltado otros elementos diferentes en el apartado de texto libre, destacando: gráficos, vídeos, poesías, imágenes, breves notas de clases, consulta con expertos, mapas conceptuales, noticias de actualidad relacionadas con la asignatura, comentarios sobre acontecimientos y perfiles de búsqueda bibliográfica.

\section{Opinión del profesorado sobre la utilidad del portafolio}

\section{El portafolio como instrumento de aprendizaje} La figura 2 permite apreciar que son muchos los factores de aprendizaje que los profesores atribuyen al portafolio. Cabe destacar que otorgan una elevada puntuación a ítems como 'potencia la reflexión del aprendiz' $(3,6)$, 'promueve el desa- 


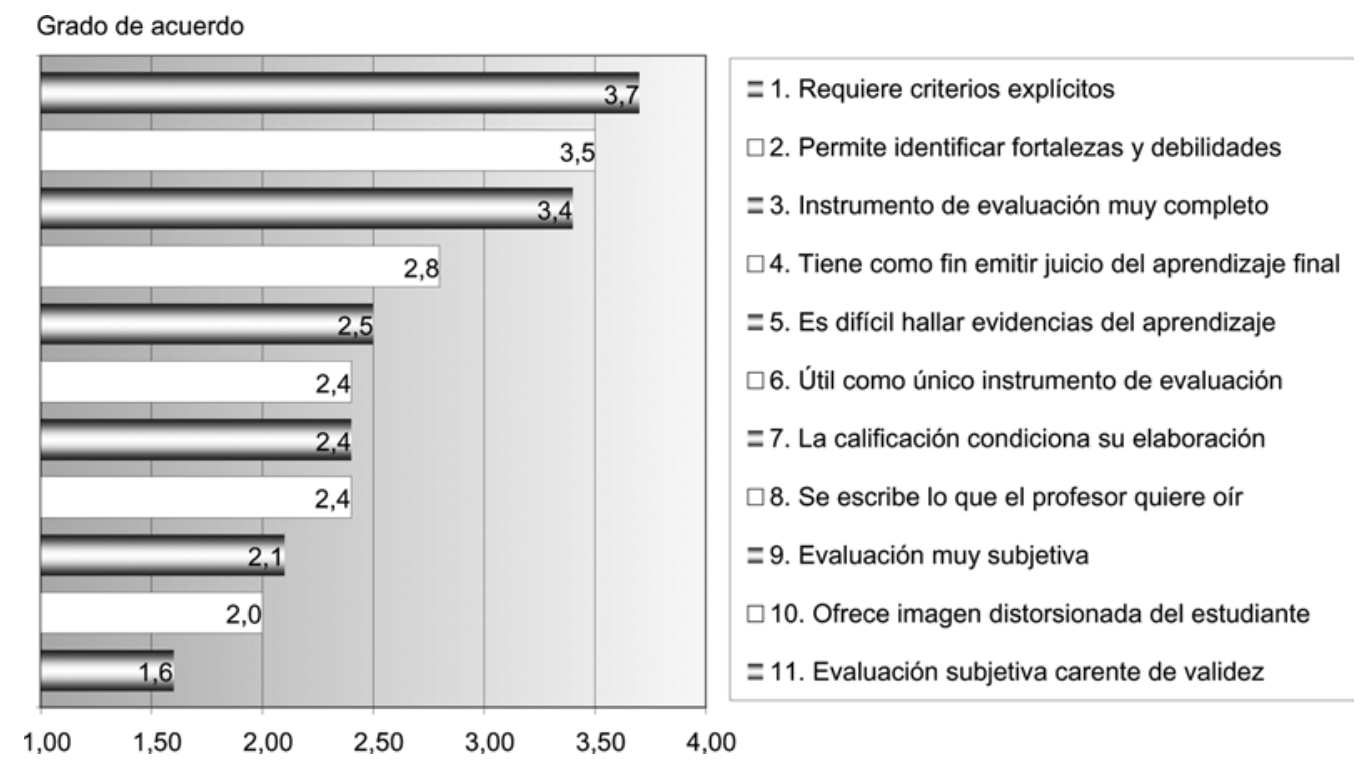

Figura 3. Opinión del profesorado sobre el portafolio como instrumento de evaluación. Escala Likert: nada, 1; poco, 2; bastante, 3; mucho, 4.

rrollo del pensamiento crítico' $(3,5)$ y 'potencia la creatividad' $(3,5)$.

\section{El portafolio como instrumento de evaluación}

Los profesores muestran un alto grado de acuerdo en considerar que la utilización del portafolio 'requiere criterios de evaluación explícitos' $(3,7)$, 'permite al estudiante la identificación de puntos fuertes y débiles' $(3,5)$ y 'constituye un instrumento de evaluación muy completo' $(3,4)$, pero no consideran que se deba utilizar como único instrumento de evaluación $(2,4)$. Asimismo hay que destacar su desacuerdo con la consideración de la evaluación del portafolio como subjetiva y carente de validez $(1,6)$ (Fig. 3).

\section{Inconvenientes del portafolio}

Los profesores consideran que la utilización de esta herramienta exige mucho tiempo de los tutores $(3,7)$, así como la preparación previa de éstos $(3,7)$ (Fig. 4).

\section{Análisis de contenido de los comentarios finales realizados en los cuestionarios}

Como se observa en la tabla, en el apartado de texto libre del cuestionario los profesores hicieron reflexiones entre las que destacan la utilidad de esta herramienta para el aprendizaje y seguimien- to de los estudiantes, la necesidad de aumentar el número de profesores, la influencia del docente en el aprendizaje de los estudiantes y la tendencia a la desaparición de los extremos en la calificación final de la asignatura al utilizar el portafolio.

\section{Discusión}

El portafolio es una herramienta que hasta la actualidad se ha utilizado en un tercio de las escuelas de enfermería españolas. Aunque en la mayoría de ellas se están realizando experiencias aisladas en algunas materias, cabe destacar que ha habido un incremento progresivo de su utilización en los últimos tres años, principalmente en los periodos de aprendizaje teórico.

Se está empleando con más frecuencia en la asignatura Fundamentos de Enfermería, lo que se puede relacionar con el hecho de que el primer artículo que se ha encontrado publicado sobre una experiencia realizada en un centro español [5] describe la introducción del portafolio en esta asignatura, lo que ha podido influir en su aplicación posterior. También es cierto que en la misma se tratan los pilares teóricos de la profesión, lo que requiere la utilización de metodologías activas que poten- 


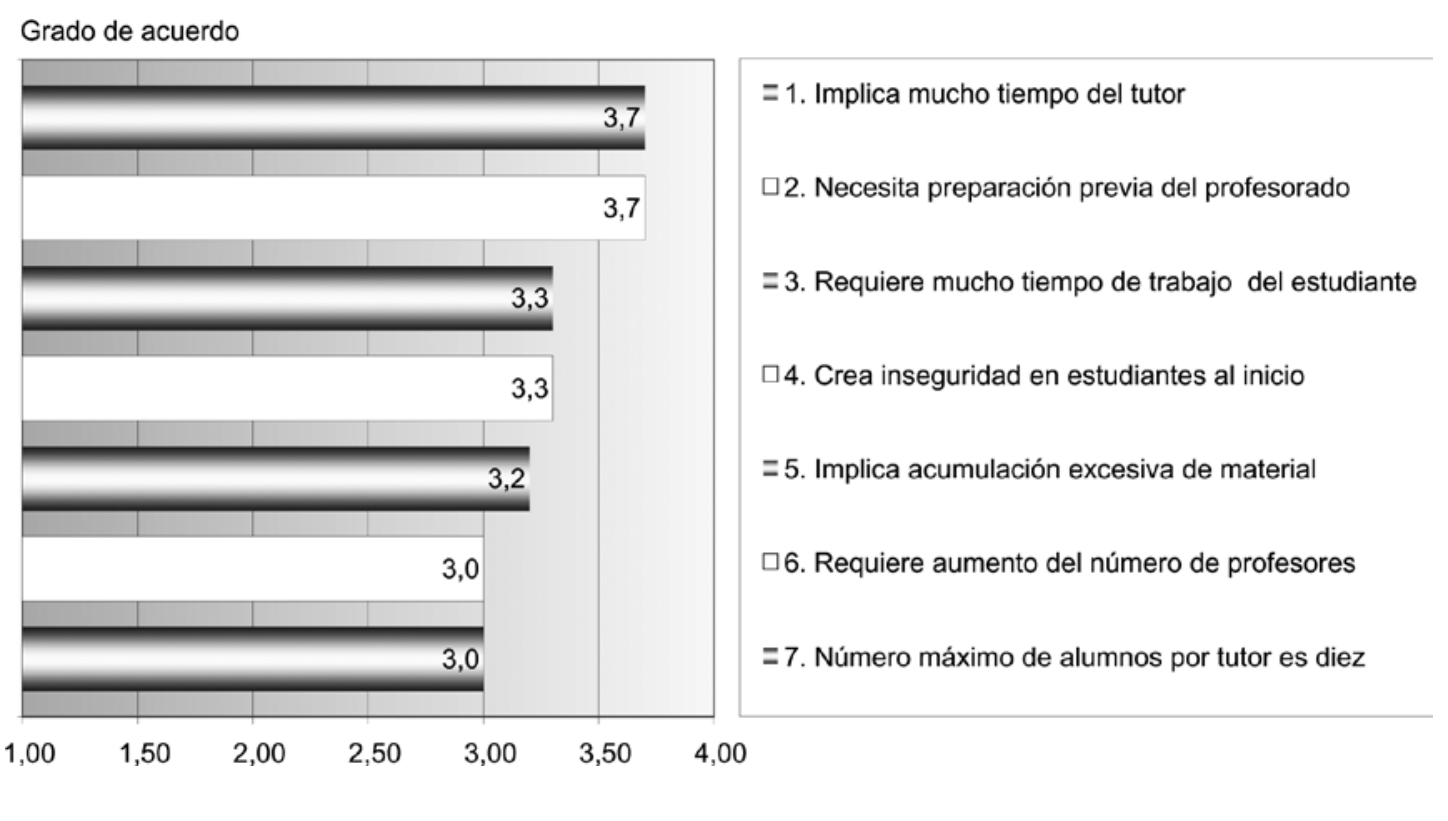

Figura 4. Opinión del profesorado sobre los inconvenientes del portafolio. Escala Likert: nada, 1; poco, 2; bastante, 3; mucho, 4.

cien el debate y la construcción del conocimiento a través de la reflexión. En este sentido estaría de acuerdo con la opinión de los estudiantes que expresan Nairn et al [10] relativa a que el portafolio es más útil para explorar el 'arte' de la profesión de enfermero que las 'habilidades técnicas'.

El profesorado participante opina que los elementos que componen el portafolio pueden ser muy variados; no obstante, al igual que McMullan [7], resaltan la escritura reflexiva como un componente esencial, lo que es coherente con la opinión mayoritaria expresada en el cuestionario sobre la contribución de dicha herramienta al desarrollo de la capacidad de reflexión del aprendiz.

Igualmente, coinciden con diversos autores $[8,11,12]$ en que la utilización del portafolio como instrumento de aprendizaje potencia el pensamiento crítico, contribuye al desarrollo de la creatividad y es una experiencia de aprendizaje significativo y profundo, que permite la integración de teoría y práctica.

Aunque consideran que es un instrumento de evaluación muy completo, en pocas ocasiones se utiliza de forma exclusiva, tendiendo a complementarlo con otras pruebas, lo que puede deberse a que se planteen dudas sobre su validez y fiabilidad para la evaluación, como señalan Kear y Bear [13], o por la dificultad de establecer los criterios cuantitativos que requiere para la calificación final de la asignatura, como afirman Webb et al [14].

Otro de los aspectos que el profesorado destaca en relación con la utilización del portafolio como instrumento de evaluación es que permite identificar los puntos fuertes y débiles del estudiante, opinión que se contrapone a la de algunos autores que consideran que el portafolio puede ofrecer una imagen distorsionada de sus debilidades y fortalezas $[15,16]$, y a la de otros que resaltan que en ocasiones el estudiante escribe lo que el profesor quiere oír [6].

No obstante, la utilización del portafolio presenta también inconvenientes, destacando, en primer lugar, que requiere mayor tiempo de trabajo del profesor [15,17] y del estudiante frente a la metodología tradicional, lo que en opinión mayoritaria del profesorado implica un aumento de plantilla; $y$, en segundo, la inseguridad que se produce en los estudiantes [2,7] al inicio de la experiencia, que podría reducirse entregándoles una guía inicial, como sugieren diversos autores [11-13,17].

Este estudio presenta limitaciones derivadas de la obtención de datos a través de un cuestionario. Se considera que se habría enriquecido notablemente con la realización de alguna entrevista al profesorado o con la obtención de muestras de 
Tabla. Análisis de contenido de los comentarios realizados por los profesores.

\begin{tabular}{|c|c|c|}
\hline Categorías & Subcategorías & $n$ \\
\hline C1. Concepto & C1.1. Enfoque diferente del portafolio entre profesores & 3 \\
\hline \multirow{6}{*}{$\begin{array}{l}\text { C2. Adquisición } \\
\text { de competencias }\end{array}$} & C2.1. Facilita la conexión de teoría y práctica & 1 \\
\hline & C2.2. Incrementa la motivación del estudiante & 1 \\
\hline & C2.3. Genera estrategias de trabajo cooperativo & 2 \\
\hline & C2.4. Potencia el aprendizaje reflexivo y crítico si hay retroalimentación del profesor & 1 \\
\hline & $\begin{array}{l}\text { C2.5. El enfoque, el trabajo y la tarea incentivadora del docente condicionan } \\
\text { la adquisición de competencias }\end{array}$ & 4 \\
\hline & C2.6. La dinámica del trabajo grupal condiciona la adquisición de competencias & 1 \\
\hline \multirow{3}{*}{$\begin{array}{l}\text { C3. Elaboración } \\
\text { del portafolio }\end{array}$} & C3.1. Necesidad de criterios compartidos con el estudiante & 2 \\
\hline & C3.2. Se debe realizar de forma voluntaria & 1 \\
\hline & C3.3. Debe contener material obligatorio que asegure el aprendizaje & 1 \\
\hline \multirow{4}{*}{ C4. Evaluación } & $\begin{array}{l}\text { C4.1. Modifica la calificación final de la asignatura, con tendencia a la desaparición } \\
\text { de los extremos }\end{array}$ & 4 \\
\hline & C4.2. Requiere una parrilla de evaluación consensuada con los alumnos & 2 \\
\hline & C4.3. Como único sistema de evaluación, exige un seguimiento continuo del estudiante & 2 \\
\hline & C4.4. Debe utilizarse junto con otras pruebas & 1 \\
\hline \multirow{3}{*}{ C5. Tutorías } & C5.1.Tienen como finalidad el feedback y la orientación del estudiante & 3 \\
\hline & C5.2. Las tutorías pueden ser grupales en seminarios & 1 \\
\hline & C5.3. La primera tutoría es fundamental para reorientar & 1 \\
\hline \multirow{5}{*}{$\begin{array}{l}\text { C6. Percepción } \\
\text { de los estudiantes }\end{array}$} & C6.1. Algunos quieren abandonar la experiencia & 1 \\
\hline & C6.2. Tienen incertidumbre al inicio y después disminuye & 2 \\
\hline & C6.3. Tienen dificultad para reflexionar & 1 \\
\hline & C6.4. Deben dedicar mucho tiempo a la lectura y elaboración & 1 \\
\hline & C6.5. Al final están satisfechos y quieren volver a utilizarlo & 3 \\
\hline
\end{tabular}


Tabla. Análisis de contenido de los comentarios realizados por los profesores (cont.).

\begin{tabular}{|c|c|c|}
\hline Categorías & Subcategorías & $n$ \\
\hline \multirow{8}{*}{$\begin{array}{l}\text { C7. Percepción } \\
\text { de las profesoras }\end{array}$} & C7.1. Experiencia positiva y gratificante & 2 \\
\hline & C7.2. Experiencia agotadora & 3 \\
\hline & C7.3. Inseguridad al principio & 1 \\
\hline & C7.4. Dificultad por la exigencia de cambio de rol & 1 \\
\hline & C7.5. La utilización del portafolio exige el aumento del número de profesores & 6 \\
\hline & $\begin{array}{l}\text { C7.6. El portafolio es una herramienta útil para el aprendizaje y seguimiento } \\
\text { de los estudiantes }\end{array}$ & 11 \\
\hline & C7.7. La utilización de esta herramienta exige cambios en la actitud del estudiante & 2 \\
\hline & C7.8. La utilización del portafolio genera cambios en el enfoque del trabajo del profesor & 3 \\
\hline \multirow{3}{*}{$\begin{array}{l}\text { C8. Apoyo } \\
\text { institucional }\end{array}$} & C8.1. Falta de proyecto común de centro & 1 \\
\hline & C8.2. Falta de recursos & 1 \\
\hline & C8.3. Para utilizar el portafolio se debe modificar la forma de distribuir a los alumnos & 1 \\
\hline
\end{tabular}

los portafolios de las diferentes experiencias y el análisis de su contenido.

Aunque en los últimos años se ha avanzado mucho en el estudio del potencial de esta herramienta en la educación de enfermeras, sería interesante seguir desarrollando líneas de investigación que profundizaran en su utilidad para impulsar el aprendizaje de competencias, principalmente en las prácticas clínicas, siguiendo el camino iniciado por otros autores [17-21], así como en la búsqueda de alternativas de mejora de la validez y fiabilidad de la evaluación que se realiza mediante esta herramienta, como afirman Byrne at al [16].

Coincidiendo con Shulman [22] hay que destacar que la construcción del portafolio es un acto teórico, ya que la teoría que cada estudiante va construyendo sobre el cuidado de las personas va a orientar la selección de evidencias que incluye en el portafolio, permitiendo descubrir las diferentes rutas que siguen los estudiantes en el aprendizaje de las competencias. Este proceso de elaboración no puede darse si no hay un cam- bio del papel tradicional que desempeñan tanto el estudiante como el profesor, de manera que es imposible sin la actividad del primero y la orientación y guía del segundo.

\section{Bibliografía}

1. Cano E. El portafolio del profesorado universitario: un instrumento para la evaluación y para el desarrollo profesional. Barcelona: Octaedro; 2005.

2. Klenowski V. Desarrollo de portafolio para el aprendizaje y la evaluación. Madrid: Narcea; 2004.

3. Martin-Kniep GO. Portafolio del desempeño de maestros, profesores y directivos. Buenos Aires: Paidós; 2001.

4. Glen S, Hight NF. Portfolios: an 'affective' assessment strategy? Nurse Educ Today 1992; 12: 416-23.

5. Olivé MC, Gusiñé F, Pardo A. Evaluación continuada en portafolio, opinión de los estudiantes referente a la ex- 
periencia llevada a cabo en la Escuela de Enfermería de la Universidad de Barcelona. Segundas Jornadas Nacionales de la Sociedad Científica Española de Licenciados en Enfermería 2004. URL: http://www.scele.enfe.ua.es/ web_scele/2_jornad.htm.

6. Scholes J, Webb C, Gray M, Endacott R, Miller C, Jasper $\mathrm{M}$, et al. Making portfolios work in practice. J Adv Nurs 2004; 46: 595-603.

7. McMullan M. Students' perceptions on the use of portfolios in pre-registration nursing education: a questionnaire survey. Int J Nurs Stud 2006; 43: 333-43.

8. Vera M, Canalejas MC. El portafolio como recurso de aprendizaje e instrumento de evaluación de estudiantes repetidores de enfermería. Educ Med 2007; 10: 114-20.

9. Spence W, El-Ansari W. Portfolio assessment: practice teachers' early experience. Nurse Educ Today 2004; 24 : 388-401.

10. Nairn S, O’Brien E, Traynor V, Williams G, Chapple M, Johnson S. Student nurses' knowledge, skills and attitudes towards the use of portfolios in a school of nursing. J Clin Nurs 2006; 15: 1509-20.

11. Bowers SP. The portfolio process: questions for implementation and practice. Coll Stud J 2005; 39: 754-8.

12. Tiwari A, Tang C. From process to outcome: the effect of portfolio assessment on student learning. Nurse Educ Today 2003; 23: 269-77.

13. Kear ME, Bear M. Using portfolio evaluation for program outcome assessment. J Nurs Educ 2007; 46: 109-13.

14. Webb CH, Endacott R, Gray MA, Jasper MA, McMullan $\mathrm{M}$, Scholes J. Evaluating portfolio assessment systems: what are the appropriate criteria? Nurse Educ Today 2003; 23: 600-9.

15. Harris S, Dolan G, Fairbairn G. Reflecting on the use of student portfolios. Nurse Educ Today 2001; 21: 278-86.

16. Byrne M, Delarose T, King CA, Leske J, Sapnas KG, Schroeter K. Continued professional competence and portfolios. J Trauma Nurs 2007; 14: 24-31.

17. Dolan G, Fairbairn G, Harris S. Is our student portfolio valued? Nurse Educ Today 2004; 24: 4-13.

18. Tracy SM, Marino GJ, Richo KM, Daly EM. The clinical achievement portfolio: an outcomes-based assessment project in nursing education. Nurse Educ 2000; 25: 241-6.

19. Williams J. The clinical notebook: using student portfolios to enhance clinical teaching learning. J Nurs Educ 2001; 40: 135-7.

20. Schaffer MA, Nelson P, Litt E. Using portfolios to evaluate achievement of population-based public health nursing competencies in baccalaureate nursing students. Nurs Educ Perspect 2005; 26: 104-12.

21. Guerra I, Mayo A, Fradejas V, Díez B. El portafolio como sistema de enseñanza-aprendizaje en las prácticas clínicas de los alumnos de la EUE de la Universidad de Valladolid. Libro de ponencias X Encuentro de Investigación en Enfermería (Investen-ISCIII), 2006. URL: http://www.isciii. es/investen.

22. Shulman L. Portafolios del docente: una actividad teórica. In Lyons N, ed. El uso del portafolios. Propuestas para un nuevo profesionalismo docente. Buenos Aires: Amorrortu; 1999. 